\title{
Effect of taxol on the expression of FoxM1 ovarian cancer-associated gene
}

\author{
ZENG LIU $^{1 *}$, YU XIAO $^{2 *}$, SIQING NING ${ }^{1}$, ZHAO YUAN LI ${ }^{1}$, YUANYUAN ZHU $^{3}$ and GANG HU ${ }^{4 *}$ \\ ${ }^{1}$ Department of Nuclear Medicine, Central Hospital of Xiangyang, Xiangyang, Hubei 441021; ${ }^{2}$ State Drug \\ Clinical Trial Agency, Sichuan Provincial People's Hospital, Sichuan Academy of Medical Science, Chengdu, \\ Sichuan 610072; ${ }^{3}$ Department of Nuclear Medicine, Xiangyang No. 1 People's Hospital, Xiangyang, Hubei 441000; \\ ${ }^{4}$ Department of Breast Surgery, Sichuan Provincial People's Hospital, Sichuan Academy \\ of Medical Science, Chengdu, Sichuan 610072, P.R. China
}

Received February 23, 2016; Accepted April 22, 2016

DOI: $10.3892 / \mathrm{ol} .2016 .4490$

\begin{abstract}
The incidence of ovarian cancer in women has been on the increase in recent years. The aim of the present study was to examine the effects of taxol on the expression of ovarian cancer-associated gene forkhead box transcription factor M1 (FoxM1) and its therapeutic effects for ovarian cancer. The expression of FoxM1 gene was examined in patients with or without ovarian cancer. RNA and protein levels of FoxM1 gene of ovarian cancer patients were detected at different time periods (1, 3, 6, 8, 12 and 24 months) after treatment with taxol. The results showed that the mRNA level of FoxM1 gene in patients with ovarian cancer was significantly higher than that in normal women $(\mathrm{P}<0.05)$. With time and progression of the disease, the expression of FoxM1 gene significantly increased in the patients not being administered taxol, whereas the expression of FoxM1 in the patients administered taxol was significantly lower comparatively $(\mathrm{P}<0.05)$. In conclusion, an asssociation was identified between the FoxM1 gene and ovarian cancer. The FoxM1 gene therefore promotes the generation and deterioration of ovarian cancer, whereas taxol reduces it. These findings provide a certain theoretical basis for the later treatment of ovarian cancer disease.
\end{abstract}

Correspondence to: Dr Yuanyuan Zhu, Department of Nuclear Medicine, Xiangyang No. 1 People's Hospital, 15 Jiefang Road, Xiangyang, Hubei 441000, P.R. China

E-mail: pmuyb0375527@163.com

Dr Gang Hu, Department of Breast Surgery, Sichuan Provincial People's Hospital, Sichuan Academy of Medical Science, Chengdu, Sichuan 610072, P.R. China

E-mail: msvec40557nja@163.com

*Contributed equally

Key words: FoxM1, ovarian cancer, RT-polymerase chain reaction, western blotting, interrelation

\section{Introduction}

The incidence of ovarian cancer in women is on the increase. Statistics obtained in 2013 by the International Anticancer Association (1) showed that ovarian cancer was associated with cervical, uterine, and breast cancer. The high incidence of these diseases accounts for $18.4 \%$ of the total number of female cancers (1). At least 137 million women are diagnosed with ovarian cancer annually, of whom approximately $32.4 \%$ succumb to ovarian cancer. The number of patients has increased significantly at an annual rate of $0.32-0.48 \%$ (1). Statistics have shown that the proportion of women with ovarian cancer in China has increased. It accounts for $17.4 \%$ of female malignant tumors, its incidence rate was approximately $26.4 \%$ and the mortality rate was approximately $35.4 \%$, slightly higher than the international average level (2). Therefore, the diagnosis and treatment of ovarian cancer has become an important research focus. Previous findings have shown that early detection and appropriate treatment modalities for ovarian cancer are essential to ensure the successful treatment of ovarian cancer $(3,4)$.

Forkhead box transcription factor M1 (FoxM1), a transcription factor identified to be associated with abnormal cell proliferation and cancer (3), was evaluated as the 'Molecule for the Year 2010' by the International Society For Molecular and Cell Biology and Biotechnology Protocols and Researches (4). He et al (5) demonstrated that FoxM1 serves as a type of transcription factor in the human body, which may activate downstream target genes regulated by it (such as signaling paths or key proteins relevant to cell proliferation) when the human body received external stimuli or had some internal changes. Wang et al (6) demonstrated that FoxM1 was closely associated with the occurrence, development and prognosis of some malignant tumors in the human body. For example, the abnormal expression of FoxM1 gene was detected in ovarian, liver and lung cancer (7). Zhang et al (8) revealed that higher FoxM1 expression level was associated with tumor prognosis, while the 5-year survival rate decreased significantly. Chen et al (9) identified that taxol had good curative effects for various types of tumors and cancer. Zhu et al (10) suggested that taxol was beneficial in the treatment of colon cancer. 
Based on these observations, the aim of the present study was ot examine the mutual association between FoxMl gene and ovarian cancer.

\section{Materials and methods}

\section{Materials}

Clinical samples. The patients with ovarian cancer and their surgical specimens were collected between 2011 and 2014 at the Sichuan Provincial People's Hospital. The patients were aged 37-54 years, with an average age of $43.5 \pm 4.6$ years. The normal controls were aged 39-55 years, with an average age of $44.3 \pm 4.2$ years. The subjects were randomly divided into the observation and control groups. The observation group included 36 women with ovarian cancer and the control group included 36 normal women.

Experimental medicines. An ovarian cancer detection kit was purchased from Roche Diagnostics (Indianapolis, IN, USA). Other drugs were purchased from Thermo Fisher Scientific, (Waltham, MA, USA). The fluorescence quantitative primers were produced by Takara Bio (Dalian, China), and FoxM1 antibody was provided by Acris Antibodies (San Diego, CA, USA).

\section{Methods}

RNA extraction of ovarian carcinoma cells. Frozen tissue samples were removed from $\sim 0.1 \mathrm{~g}$ liquid nitrogen, thawed on ice and $0.45 \mathrm{ml}$ RNA Plus (Beijing Ed Biological Technology Co, Ltd., Beijing, China) was added. Subsequently, the tissues were homogenized and $0.45 \mathrm{ml}$ of RNA Plus was added. Chloroform (200 $\mu$ l) was then added and briefly mixed followed by centrifugation at $8,000 \mathrm{x} \mathrm{g}$ for $15 \mathrm{~min}$ at $<4^{\circ} \mathrm{C}$. The supernatant was transferred into an Eppendorf tube (Beijing Ed Biological Technology Co, Ltd.) with an equal volume of isopropanol and mixed, prior to centrifugation at $8,000 \mathrm{xg}$ for $10 \mathrm{~min}$ at $<4^{\circ} \mathrm{C}$. The supernatant was removed, $750 \mu 1$ ethanol $(75 \%)$ was added to mix gently, and the mixture was centrifuged at $8,000 \mathrm{x}$ g for $10 \mathrm{~min}$ at $4^{\circ} \mathrm{C}$. The supernatant and the remainder of the residual ethanol were subsequently removed. An appropriate amount of RNase-free water was added to the RNA pellet.

Fluorogenic quantitative polymerase chain reaction ( $q P C R)$. Fluorogenic quantitative polymerase chain reaction was conducted according to the protocol for Takara Bio fluorescence qPCR.

Detection of FoxM1 expression in serum with ELISA. ELISA was carried out according to the manufacturer's instructions (11). The samples were diluted at a ratio of 1:200, and $100 \mu 1$ serum samples were added into each well. FoxM1 detection solution was then added into the wells with $50 \mu \mathrm{l} /$ well at $25^{\circ} \mathrm{C}$ for $1.5 \mathrm{~h}$. TMB substrate $(50 \mu \mathrm{l})$ was then added into each well for color development. The optical density was measured at $495 \mathrm{~nm}$ using a fluorescent plate reader (Hewlett-Packard Development Company, Palo Alto, USA) to calculate FoxM1 expression for each sample by comparing with the standard curve.

Detection of FoxM1 in ovarian cancer tissues with immunohistochemistry (IHC). IHC for ovarian tissue samples was performed according to the streptomycin affinity peroxidase

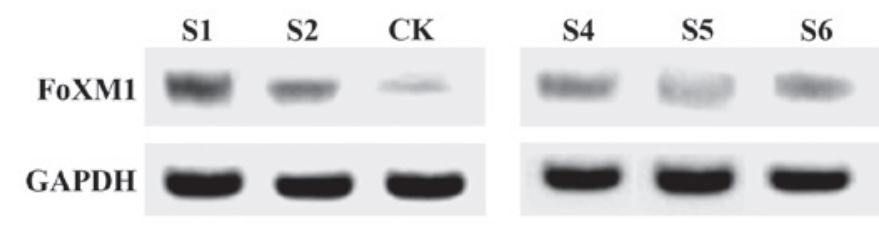

Figure 1. The FoxM1 expression of the normal women and patients with ovarian cancer. CK, FoxM1 expression in the normal women; S1-S6, FoXM1 expression in patients at different time points.

(S-P) method (12). Staining was calculated as follows: $<10 \%$ or negative, negative (-); only stained cell membrane or $>10 \%$ tumor cells, weak positive (+); $>10 \%$ tumor cells indicated weak or moderately complete staining, medium strong positive $(++)$; and $>10 \%$ tumor cells indicated markedly complete membrane staining, strong positive (+++).

Detection of FoxM1 in ovarian cancer tissues and serum using western blotting. A Thermo Fisher Scientific animal cell protein extraction kit was used to extract the total protein in the samples (particular operation according to the specification) (13). Western blotting was conducted as previously described (14). The primary antibody was anti-FoxM1 (rabbit polyclonal antibody, diluted 1:500, cat.no: sc-502; Santa Cruz Biotechnology, Inc., Santa Cruz, CA, USA) and secondary antibody was horseradish peroxidase-conjugated anti-rabbit IgG (diluted 1:5,000; Santa Cruz Biotechnology, Inc., Santa Cruz, CA, USA).

Statistical analysis. SPSS 20.0 software (IBM SPSS, Armonk, NY, USA) was used to for the statistical analysis. The measurement data were presented as mean \pm standard deviation, while $\chi^{2}$ test was used for the count data.

\section{Results}

mRNA expression of FoxM1 in patients with or without ovarian cancer. In the present study, we selected ovarian tissue samples of normal women and ovarian cancer patients. The RNA was extracted from the tissue samples for fluorescence qPCR as mentioned in Materials and methods. The primer sequences used were : Forward 5'-TTTTGCTAGCTC AAGCCCTGTCAACTTTACC-3', and reverse 5'-ATATAA GCTTTTGCTGCATCCCGCTCACCT-3'. Fig. 1 shows the electrophoretic result of the PCR products. FoxM1 mRNA content in patients with ovarian cancer was higher than that in the normal population (CK), and its expression was not the same at the different time points. By comparing the expression of FoxM1 mRNA in the experimental and control groups (Fig. 2) the average level of FoxM1 mRNA expression in patients with ovarian cancer was found to be 4.3- to 5.8-fold significantly higher than that in the normal women. The result identified a certain correlation between FoxMI gene and ovarian cancer.

FoxM1 mRNA expression in ovarian cancer patients at different stages. FoxM1 mRNA expression was detected in ovarian cancer patients at different time points (Fig. 3). The expression of FoxM1 gene gradually increased with the 


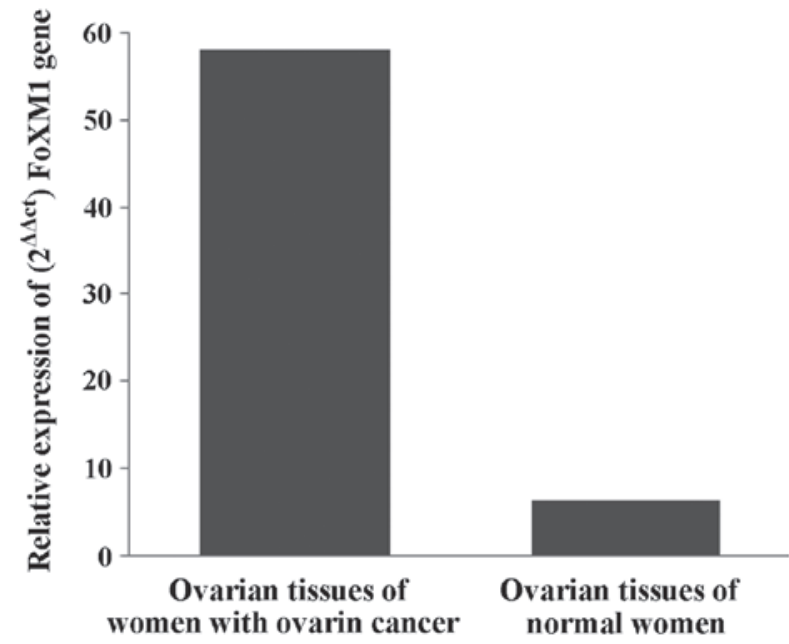

Figure 2. The relative expression of FoxM1 mRNA in the observation and control groups.

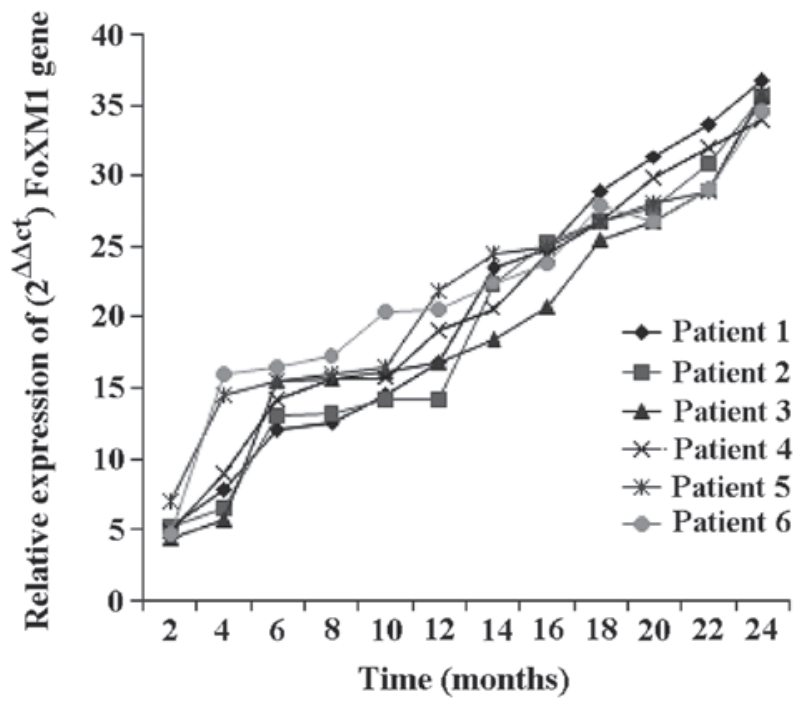

Figure 3. Changes of FoxM1 expression at different time points in different ovarian cancer patients (Patients 1-6).

progression of disease, the growth was gradual at the early stage of disease and became rapid at the later stage (6 months later). Within 8-18 months of diagnosis of ovarian cancer, the mRNA expression increased, indicating that FoxMl gene is associated with ovarian cancer, and there is a positive correlation between the levels of FoxM1 and the severity of ovarian cancer patients.

Expression of FoxM1 mRNA in serum of ovarian cancer patients at different stages. FoxM1 protein expression in the serum of ovarian cancer patients at different time points was detected using western blotting (Fig. 4). The results showed that the expression level of FoxM1 protein in serum gradually increased as the disease progressed and the growth was significantly increased after six months (Fig. 3). The results showed that FoxM1 gene is associated with ovarian cancer, and there is a positive correlation between the expression levels of FoxM1 in serum and the severity of ovarian cancer patients.

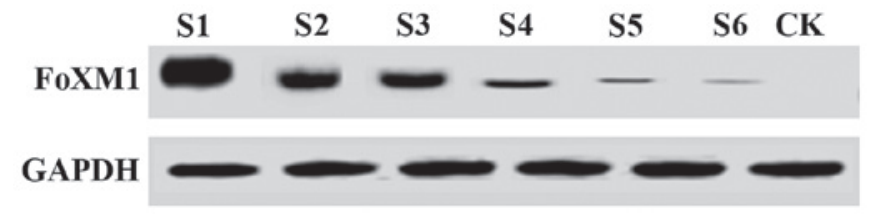

Figure 4. FoxM1 expression at different time points of one ovarian cancer patient. CK, serum of normal women; 1-6, serum of patients suffering from ovarian cancer at $24,12,8,6,3$ and 1 month.
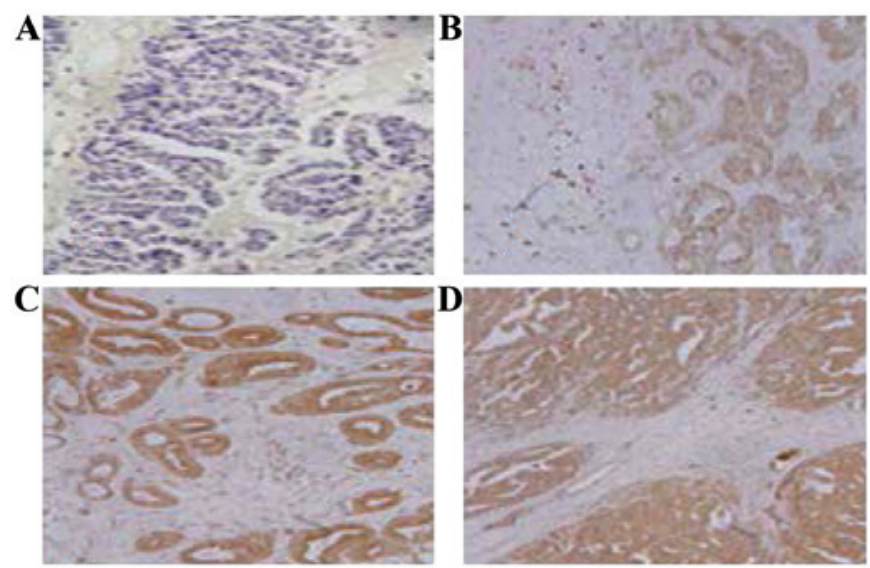

Figure 5. Immunohistochemical staining observation results of FoxM1 in human ovarian cancer tissues. (A) normal ovarian tissues (x200); (B) ovarian cancer tissues (x200); (C) ovarian cancer tissues (x200); (D) ovarian cancer tissues (x200).

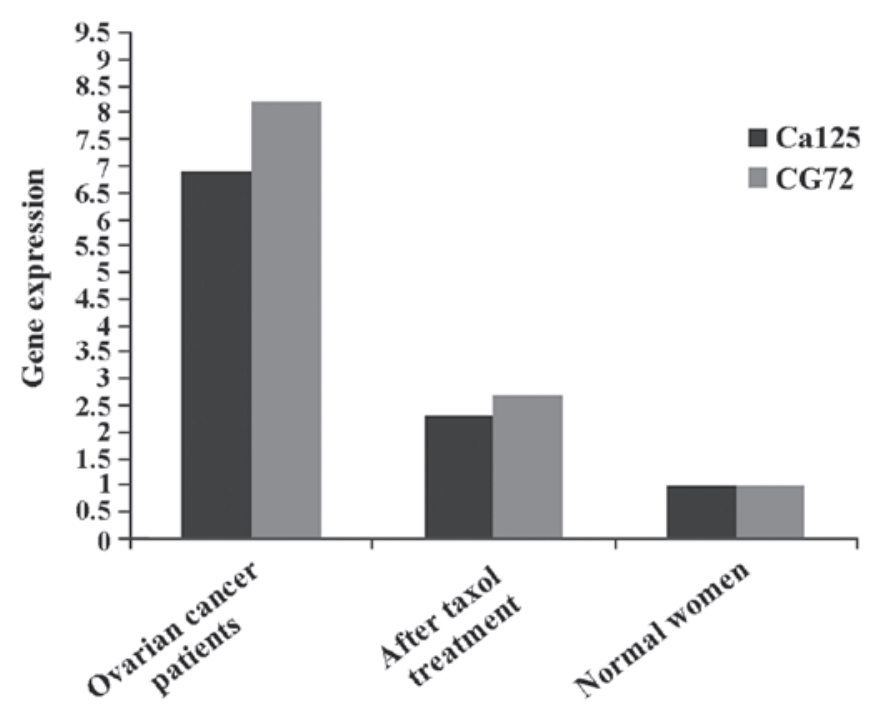

Figure 6. Recovery of ovarian cancer patients prior to and following taxol treatment.

IHC results of FoxM1 in ovarian cancer tissues (Fig. 5) demonstrated that positive staining of FoxMl expression was mainly concentrated in the ovarian cancer cell membrane (B-D), and the expression was shown as: i) Negative (-); ii) weak positive (+); iii) moderately strong positive (++); and iv) strong positive $(+++)$. The main features of positive staining were the uneven size of brown small particles, while there were not 
found in the normal ovarian tissues. The content of FoxM1 in ovarian cancer patients was higher than that in the normal population and mainly concentrated in the ovarian tissue.

Curative effects of taxol on ovarian cancer patients. In the present study, we preliminarily examined the curative effects of taxol on ovarian cancer patients. The results revealed corresponding sensitive markers of ovarian cancer (CA125, AG72), and contents of the above sensitive markers in ovarian cancer patients of the experimental group administered taxol significantly decreased, suggesting that taxol has curative effects on ovarian cancer to a certain extent (Fig. 6).

Expression of FoxM1 in ovarian cancer patients prior to and following taxol treatment. In the present study, the patients with ovarian cancer were treated with taxol. Prior to and following therapy, the expression of FoxM1 in the lesion tissues of the experimental samples was measured by ELISA, and results revealed that the average content of FoxM1 in the serum of normal women was approximately $4.19 \pm 0.63 \mathrm{ng} / \mathrm{ml}$. The results for the 36 female ovarian cancer patients revealed that the average content of FoxM1 in the serum prior to treatment was $12.1 \pm 21.21 \mathrm{ng} / \mathrm{ml}$, and that after treatment with taxol the serum level was approximately $5.73 \pm 0.39 \mathrm{ng} / \mathrm{ml}$. The content in the serum of ovarian cancer patients $(12.12 \pm 1.21 \mathrm{ng} / \mathrm{ml})$ was significantly higher than that in the serum of the controls $(4.19 \pm 0.63 \mathrm{ng} / \mathrm{ml})$. Compared with conditions prior to treatment, the expression of FoxM1 in ovarian cancer patients treated with taxol was significantly decreased, indicating that taxol exerts certain effects on ovarian cancer. Therefore, the taxol may be used to treat ovarian cancer by reducing the FoxM1 gene expression in patients.

\section{Discussion}

Previous findings by Francis et al (15) revealed that FoxM1 is an important transcription factor, and is closely associated with tumor occurrence and development. The study by Yoshida et al (16) demonstrated that FoxM1 comprised 10 exons located at 12 p13-3 positions of the $20 \mathrm{~kb}$ chromosome. The expression amount of FoxM1 genes in cancer cells has been found to be significantly higher than that in the normal cells, and it can be taken as a marker for certain types of tumor and cancer (16). The percentage of female ovarian cancer in China has increased annually $(17,18)$, and the rapid diagnosis of ovarian cancer has become a hot research topic. Chen et al (19) suggested that genes associated with the occurrence and deterioration of ovarian cancer can be classified as those associated with ovarian cancer at the mRNA level and those associated with ovarian cancer at the protein level. Gong et al (20) identified 61 genes possibly associated with ovarian cancer, although those authors did not examine the mechanism involved in mRNA and protein expression levels in a detailed manner.

Taxol was identified in 1971 by Wani et al (21) who separated it from the bark of short-leaf taxus brevifolia. Wang et al (22) previously demonstrated that it taxol may serve as a broad-spectrum antitumor drug. Schiff et al proved that taxol had a unique anticancer mechanism $(23,24)$. For instance, it acted on cell microtubules and to a certain extent induced protein separation in relevant proteins by interplaying with the amino acid at the 31st position at the $\mathrm{N}$ end of the microtubule and the amino acid at the 217th-231st position. Further blocking cells in the G2PM period and ultimately causing abnormality or ceasing of mitosis, apoptosis of cancer cells, owing to the inability of the cells to multiply, can be used for treatment of the cancer (24). In the present study, we found that FoxM1 gene was associated with the onset of some tumors and cancer, and demonstrated that it was correlated with ovarian cancer. The expression of FoxM1 in ovarian cancer patient serum and ovarian tissue was significantly higher than that in normal women. To the best of our knowledge, for the first time, we confirmed by experiment that, taxol also had certain therapeutic effects on female ovarian cancer. Compared with conditions prior to treatment, contents of relevant sensitive markers (CA125 and AG72) of ovarian cancer in patients decreased significantly, By comparing the expression amount of FoxM1 gene in ovarian cancer patients prior to and following treatment with taxol, we found that the expression amount of FoxM1 gene significantly decreased following treatment.

In conclusion, we preliminarily evaluated treatment of female ovarian cancer with taxol, and subsequently, to the best of our knowledge, identified a new gene, FoxM1, which was associated with female ovarian cancer. By comparing the expression amount of FoxMl gene in the observation and control groups, it was found that in comparison to the normal women, the FoxM1 levels in patients with ovarian tumor significantly increased $(\mathrm{P}<0.05)$. Additionally, the results showed that FoxM1 gene expression was reduced in ovarian cancer patients after treatment with taxol. Thus, the curative effects of female ovarian cancer were preliminarily evaluated. Compared with the control group without taxol treatment, contents of relevant sensitive markers of ovarian cancer (CA125 and AG72) in ovarian patients decreased significantly, suggesting that taxol exerted a therapeutic effect on ovarian cancer. Measuring the expression amount of FoxM1 gene in ovarian cancer patients prior to and following taxol treatment showed that the expression of FoxM1 with taxol significantly decreased ovarian cancer patients. The finding suggests that taxol is capable of reducing FoxM1 levels in ovarian cancer patients. Therefore, taxol has promising implications for the treatment of ovarian cancer and may be developed as a therapeutic agent.

\section{References}

1. Zheng RS, Zhang SW, Wu LY, Li GL, Zhao P, Hao J and Chen WQ: Report of incidence and mortality from China cancer registries in 2008. China Cancer 21: 1-12, 2012.

2. Pei GJ, Fu L, Cui YL, Gao LH, Wang WL and Lu WQ: Meta-analysis of risk factors of breast cancer in Chinese women. China Cancer 21: 1-12, 2012.

3. Laoukili J, Stahl M and Medema RH: FoxM1: At the crossroads of ageing and cancer. Biochim Biophys Acta 1775: 92-102, 2007.

4. Yap KL, Fraley SI, Thiaville MM, Jinawath N, Nakayama K, Wang J, Wang TL, Wirtz D and Shih IeM: NAC1 is an actin-binding protein that is essential for effective cytokinesis in cancer cells. Cancer Res 72: 4085-4096, 2012.

5. He SY, Shen HW, Xu L, Zhao XH, Yuan L, Niu G, You ZS and Yao SZ: FoxM1 promotes tumor cell invasion and correlates with poor prognosis in early-stage cervical cancer. Gynecol Oncol 127: 601-610, 2012. 
6. Wang Z, Ahmad A, Li Y, Banerjee S, Kong D and Sarkar FH: Forkhead box M1 transcription factor: A novel target for cancer therapy. Cancer Treat Rev 36: 151-156, 2010.

7. Yeasmin S, Nakayama K, Rahman MT, Rahman M, Ishikawa M, Katagiri A, Iida K, Nakayama N, Otuski Y, Kobayashi H, et al: Biological and clinical significance of NAC1 expression in cervical carcinomas: A comparative study between squamous cell carcinomas and adenocarcinomas/adenosquamous carcinomas. Hum Pathol 43: 506-519, 2012.

8. Zhang Y, Cheng Y, Ren X, Hori T, Huber-Keener KJ, Zhang L, Yap KL, Liu D, Shantz L, Qin ZH, et al: Dysfunction of nucleus accumbens-1 activates cellular senescence and inhibits tumor cell proliferation and oncogenesis. Cancer Res 72: 4262-4275, 2012.

9. Chen YZ, Chen KZ and Zeng JJ: Observation and nursing of taxol in the treatment of cancer chemotherapy. J Qilu Nurs 15: 49-50, 2009 (In Chinese).

10. Zhu HC, Zhao BZ, Zheng XZ and Zhu YT: The effect of taxol on the expression of Bcl-2 and Bax in colon carcinoma HT-29 cells. Chin Med Mod Dist Educ Chin 10: 141-142, 2012 (In Chinese).

11. Zhang Y, Cheng Y, Ren X, Zhang L, Yap KL, Wu H, Patel R, Liu D, Qin ZH, Shih IM and Yang JM: NAC1 modulates sensitivity of ovarian cancer cells to cisplatin by altering the HMGB1-mediated autophagic response. Oncogene 31: 1055-1064, 2012.

12. Okazaki K, Nakayama N, Nariai Y, Nakayama K, Miyazaki K, Maruyama R, Kato H, Kosugi S, Urano T and Sakashita G: Nuclear localization signal in a cancer-related transcriptional regulator protein NAC1. Carcinogenesis 33: 1854-1862, 2012.

13. Wu PH, Hung SH, Ren T, Shih IeM and Tseng Y: Cell cycle-dependent alteration in NAC1 nuclear body dynamics and morphology. Phys Biol 8: 015005, 2011.

14. Padda RS, Gkouvatsos K, Guido M, Mui J, Vali H and Pantopoulos K: A high-fat diet modulates iron metabolism but does not promote liver fibrosis in hemochromatotic $\mathrm{Hjv}^{-/}$mice. Am J Physiol Gastrointest Liver Physiol 308: G251-G261, 2015.
15. Francis RE, Myatt SS, Krol J, Hartman J, Peck B, McGovern UB, Wang J, Guest SK, Filipovic A, Gojis O, et al: FoxM1 is a downstream target and marker of HER2 overexpression in breast cancer. Int J Oncol 35: 57-68, 2009.

16. Yoshida Y, Wang IC, Yoder HM, Davidson NO and Costa RH: The forkhead box M1 transcription factor contributes to the development and growth of mouse colorectal cancer. Gastroenterology 132: 1420-1431, 2007.

17. Yan XD and Pan LY: Comparative proteomic analysis of platinum drug resistance related proteins in ovarian cancer and the research on the function of drug resistance related protein Annexin A3. Chin J Obstet Gynecol 41: 584-587, 2006 (In Chinese).

18. Sun P and Bai P: An analysis of prognostic factors in 32 cases with transitional cell carcinoma of the ovary. J Chin Oncol 17: 97-100, 2011 (In Chinese).

19. Chen LL: Ovarian epithelium carcinoma induce abnormal differentiation of dendritic cell precursors (unpublished $\mathrm{PhD}$ thesis). Zhejiang University 2009.

20. Gong YQ, Han FJ and Wu XK: Advances in etiology of epithelial ovarian cancer. J Med Res: 18-20, 2010.

21. Wani MC, Taylor HL, Wall ME, Coggon P and McPhail AT: Plant antitumor agents. VI. The isolation and structure of taxol, a novel antileukemic and antitumor agent from Taxus brevifolia. J Am Chem Soc 93: 2325-2327, 1971.

22. Wang ZH, Zeng HX and Chang XH: A experiment study of effects on glycometabolism after the application of cortisol in chemotherapy includes taxoll. Prog Obstet Gynecol 19: 206-209, 2010 (In Chinese)

23. Schiff PB, Fant J and Horwitz SB: Promotion of microtubule assembly in vitro by taxol. Nature 277: 665-667, 1979.

24. Schiff PB and Horwitz SB: Taxol stabilizes microtubulesin mouse fibroblast cells. Proc Natl Acad Sci USA 77: 1561-1565, 1980. 